\title{
Noise suppression due to long-range Coulomb interaction: Crossover between diffusive and ballistic transport regimes
}

\author{
T. González, O. M. Bulashenko, J. Mateos, and D. Pardo \\ Departamento de Física Aplicada, Universidad de Salamanca, Plaza de la Merced s/n, \\ E-37008 Salamanca, Spain \\ L. Reggiani \\ Istituto Nazionale di Fisica della Materia, Dipartimento di Scienza dei Materiali, \\ Università di Lecce, Via Arnesano, 73100 Lecce, Italy \\ J. M. Rubí \\ Departament de Física Fonamental, Universitat de Barcelona, Av. Diagonal 647, \\ E-08028 Barcelona, Spain \\ (10 February 1997) \\ (cond-mat/9703123, Semicond. Science and Technol., v.12, 1053 (1997))
}

\begin{abstract}
We present a Monte Carlo analysis of shot-noise suppression due to long-range Coulomb interaction in semiconductor samples under a crossover between diffusive and ballistic transport regimes. By varying the mean time between collisions we find that the strong suppression observed under the ballistic regime persists under quasi-ballistic conditions, before being washed out when a complete diffusive regime is reached.
\end{abstract}

It has been recognized that shot noise and thermal noise are not different sources of noise, but just special limits of a more general concept (see discussion by Landauer [1]), which can be treated in a unified way [1,2]. This point has been clarified by studying noise properties of mesoscopic systems such as quantum point contacts, quantum wires, quantum dots, etc [3] in which both ballistic $\left(\lambda_{p} \gg L\right)$ and diffusive $\left(\lambda_{p} \ll L\right)$ regimes of carrier transport are accessible (here $\lambda_{p}$ is the mean free path and $L$ is the device length).

A matter of particular interest is the suppression of shot noise resulting from the correlation among carriers in their transmission through a device (see some recent experiments 4, 4, 6, 6,8 and the status of theoretical developments [3]). Among the different mechanisms of noise suppression most attention has been paid to the correlations imposed by the Pauli principle (Fermi statistics) under degenerate conditions. In this case, the reduction of noise has been found for both ballistic [9] and diffusive regimes [10], and the crossover between them has been studied theoretically by de Jong and Beenakker [11].

However, as emphasized by Landauer [1], apart from Pauli correlations, Coulomb interaction between carriers may also be a source of noise suppression. By using an ensemble Monte Carlo simulator self-consistently coupled with a Poisson solver (PS) we have calculated the shot-noise suppression associated with long-range Coulomb interaction in the case of perfect ballistic transport [12]. Here we address the case of low-density carrier concentration typical of semiconductors, thus avoiding any effect due to statistical degeneracy. Under this condition we have found that this suppression is stronger as space-charge effects become more important, and can achieve higher levels for increasing values of the parameter $\lambda=L / L_{D c}$, where $L_{D c}=\sqrt{\varepsilon k_{B} T / q^{2} n_{c}}$ is the Debye length corresponding to the carrier concentration at the injecting contact ( $T$ is the lattice temperature, $k_{B}$ is Boltzmann constant, $\varepsilon$ is the dielectric constant, $q$ is the electron charge, and $n_{c}$ is the carrier concentration at the contact). Therefore, the longer the device length, the larger the shot-noise suppression is expected to be, provided that transport remains ballistic. However, with increasing the device length (or lattice temperature) carrier transport actually becomes diffusive and the disappearance of noise suppression associated with Coulomb correlation is expected. Note that in the case of metals all charge fluctuations are screened out and Coulomb correlations play no role in the dc transport regime. It is the aim of the present letter to investigate the behavior of noise suppression due to the long-range Coulomb interaction among carriers in the crossover region from ballistic to diffusive transport regimes in nondegenerate semiconductors. We will show that noise suppression still remains important under quasi-ballistic conditions, before being washed out by the action of scattering mechanisms.

For the calculations we consider the following simple model: a lightly doped active region of a semiconductor sample sandwiched between two heavily doped contacts (of the same semiconductor) injecting carriers into the active region. The contacts are considered to be ohmic (the voltage drop inside them is negligible) and they remain always in thermal equilibrium. Thus electrons are emitted from the contacts according to a thermalequilibrium Maxwell-Boltzmann distribution and they move inside the active region, following the semiclassical equations of motion, by undergoing elastic and isotropic scattering. The fluctuating injection rate at the contacts, which is associated with the diffusion current of a homojunction, is taken to follow a Poissonian statistics. Accordingly, the time between two consecutive electron injections is generated with a probability per unit time $P(t)=\Gamma e^{-\Gamma t}$, where $\Gamma=\frac{1}{2} n_{c} v_{\mathrm{th}} S$ is the injection rate, $v_{\mathrm{th}}=\sqrt{2 k_{B} T / \pi m}$ is the thermal velocity, $S$ is the crosssectional area of the device and $m$ is the electron effective mass. The simulation is one-dimensional in real space (Poisson's equation is solved only in one dimension) and three-dimensional in momentum space.

For the simulations we have used the following set of 
parameters: $T_{0}=300 \mathrm{~K}, m=0.25 m_{0}$ ( $m_{0}$ being the free electron mass), relative dielectric constant $\varepsilon=11.7$, $L=2000 \stackrel{\circ}{A}$ and $n_{c}=4 \times 10^{17} \mathrm{~cm}^{-3}$, much higher than the sample doping (here taken of $10^{11} \mathrm{~cm}^{-3}$, but the same results are obtained up to $10^{15} \mathrm{~cm}^{-3}$ ). The above set of values yields for the dimensionless parameter $\lambda=L / L_{D c}$, which characterizes the importance of the electrostatic screening, the value $\lambda=30.9$. Along with $\lambda$, we introduce the dimensionless ballistic parameter $\ell=v_{\mathrm{th}} \tau / L$ which characterizes the crossover between diffusive and ballistic transport regimes. The average time between collisions in the bulk $\tau$ is assumed to be independent of energy [13], and it is varied from $10^{-15} \mathrm{~s}$ to $10^{-11} \mathrm{~s}$, so that both regimes of carrier transport, ballistic $(\ell \gg 1)$ and diffusive $(\ell \ll 1)$, are covered. For the PS, a time step of 2 fs and 100 meshes in real space are taken.

We apply a $d c$ voltage and calculate the time-averaged current $I$ and the current autocorrelation function $C_{I}(t)$ by means of the ensemble Monte Carlo simulator selfconsistently coupled with the PS [14]. We stress that in our approach the number of electrons $N$ inside the sample is not required to be fixed. Carriers are injected at $x=0$ and $x=L$ inside the active region of the device according to $P(t)$. When a carrier exits through any of the contacts it is cancelled from the simulation statistics, which accounts only for the carriers which are inside the device at the given time $t$. Thus, $N(t)$ fluctuates in time due to the random injection from the contacts and we can evaluate both the time-averaged value $\langle N\rangle$ and its fluctuations $\delta N(t)=N(t)-\langle N\rangle$. To illustrate the importance of the effects associated with the long-range Coulomb interaction, we provide the results for two different simulation schemes. The first one involves a dynamic PS, so that any fluctuation of space charge arising because of the random injection from the contacts causes a redistribution of the potential which is self-consistently updated by solving the Poisson equation at each time step during the simulation. The second scheme makes use of a static PS, so that only the stationary potential profile is calculated; once the steady state is reached, the PS is switched off and carriers move in the frozen non-fluctuating electric field profile. Both schemes give exactly the same average current and steady-state spatial distributions of all the quantities, but the noise characteristics are quite different.

By varying the ballistic parameter $\ell$ through $\tau$ we find that the steady-state spatial profile of the potential does not change significantly. It always exhibits a minimum near the cathode due to the presence of space charge whenever the current does not saturate. This minimum acts as a potential barrier for the electrons moving between the contacts, so that those electrons which do not have enough energy to pass over the barrier are reflected back to the contacts. The most important fact is that the barrier height and, as a consequence, the transmission through it depends on the current, which is crucial in calculating the noise characteristics.

Fig. 1 presents the results for the low-frequency spectral density of current fluctuations $S_{I}=2 \int_{-\infty}^{\infty} C_{I}(t) d t$ as a function of $\ell$ for an applied voltage of $U=40 k_{B} T / q$.
For the present value of $\lambda$ this voltage corresponds to the maximum suppression of noise, reaching a value of 0.045 . The results are normalized to $2 q I_{s}$, where $I_{s}=q \Gamma=\frac{1}{2} q n_{c} v_{\mathrm{th}} S$ is the saturation current (maximum current that our contacts in thermal equilibrium can provide). Both static and dynamic PS cases are shown. The curve $2 q I$, reported for comparison, illustrates how the average current changes with the ballistic parameter $\ell$. In the diffusive regime $(\ell \rightarrow 0)$ the current decreases linearly with $\ell$ (or, which is the same, with $\tau$ ) since scattering processes prevent carriers from gaining velocity in the field direction [15]. The noise in this regime corresponds to thermal (nonequilibrium) noise, and thus $S_{I}$ is also proportional to $\ell$. Both PS schemes provide identical results, which means that the action of the scattering mechanisms prevails over that of the self-consistent field fluctuations. When approaching the ballistic regime, the current increases sublinearly with $\ell$ until it saturates at the value $0.65 I_{s}$ in the ballistic limit $(\ell \rightarrow \infty)$. Starting from $\ell=0.01$ the noise departs from the thermal behavior. In the static case it obeys the classical formula $2 q I$ typical of full shot noise (Poissonian statistics, no correlation among carriers is taken into account). On the contrary, in the dynamic case $S_{I}$ is strongly reduced with respect to $2 q I$ as $\ell$ increases due to the correlations associated with the self-consistent potential. A suppression factor of more than one order of magnitude $(0.045)$ is obtained in the ballistic limit. In the intermediate quasiballistic region the dynamic noise is observed to exhibit a maximum value at $\ell \approx 0.1$. Then, the noise spectral density decreases with increasing $\ell$, which is somewhat surprising in view of a corresponding increase of the current. Notice that the difference between the two schemes starts to appear already at $\ell \sim 0.01$, which means that the long-range Coulomb interactions influence the noise even for almost "diffusive" regimes when an electron undergoes $\sim 10^{2}$ scattering events while crossing the active region of the device. Another remarkable fact is that $S_{I}$ in the static case already follows the $2 q I$ law for $\ell=0.03$, although under such conditions a carrier undergoes about 30 scattering processes in its transfer between contacts.

To clarify the role played by different fluctuating mechanisms in giving the total noise we decompose the current autocorrelation function into three contributions $C_{I}(t)=C_{V}(t)+C_{N}(t)+C_{V N}(t)$, respectively given by

$$
\begin{aligned}
C_{V}(t) & =\frac{q^{2}}{L^{2}}\langle N\rangle^{2}\left\langle\delta v\left(t^{\prime}\right) \delta v\left(t^{\prime}+t\right)\right\rangle \\
C_{N}(t) & =\frac{q^{2}}{L^{2}}\langle v\rangle^{2}\left\langle\delta N\left(t^{\prime}\right) \delta N\left(t^{\prime}+t\right)\right\rangle \\
C_{V N}(t) & =\frac{q^{2}}{L^{2}}\langle v\rangle\langle N\rangle\left\langle\delta v\left(t^{\prime}\right) \delta N\left(t^{\prime}+t\right)\right. \\
& \left.+\delta N\left(t^{\prime}\right) \delta v\left(t^{\prime}+t\right)\right\rangle
\end{aligned}
$$

in the above equations $C_{V}$ is associated with fluctuations in the mean carrier velocity $\delta v(t)=v(t)-\langle v\rangle, C_{N}$ with fluctuations in the carrier number and $C_{V N}$ with their cross-correlation [14]. The spectral densities of the current fluctuations corresponding to these contributions are illustrated in Fig. 22. For $\ell \rightarrow 0$, i.e. in the thermal 
noise limit, $S_{N} \rightarrow 0, S_{V N} \rightarrow 0$ and $S_{I} \rightarrow S_{V}$, which means that velocity fluctuations are the main source of noise. Here $S_{V}$ is proportional to $\tau$, thus corresponding to a diffusive behavior. It should be noted that the difference in the velocity-fluctuation terms $S_{V}$ for the two schemes is practically negligible in the whole range of values taken by the ballistic parameter $\ell$ [Fig. 2(a)]. On the contrary, for the other two contributions the difference becomes dramatic starting from $\ell \approx 0.03$. The number fluctuations increase with the ballistic character of transport. However such an increase is much more pronounced in the static case than in the dynamic case where, under the action of the self-consistent potential fluctuations, number fluctuations are significantly suppressed. The velocity-number correlations, represented by $S_{V N}$, show an opposite behavior. Their contribution is positive in the static case, while it is negative in the dynamic case. Furthermore, for the current spectral densities calculated in the dynamic scheme, $S_{N}$ and $S_{V N}$ are of opposite sign thus practically compensating each other. As a consequence, the current noise is considerably suppressed in the dynamic case with respect to the static case. This result reflects the fact that as carriers move ballistically (or quasi-ballistically) through the active region, the dynamic fluctuations of the electric field modulate the transmission through the potential minimum and smooth out the current fluctuations (of shot-noise type) imposed by the random injection at the contacts. The presence of scattering mechanisms, by randomizing the carrier velocity, reduces the fluctuations associated with carrier number before washing them out completely when the carrier motion is fully diffusive, leading the current noise to become thermal.

In conclusion, within a Monte Carlo scheme we have investigated the influence of long-range Coulomb interaction on shot-noise suppression in the transition region from ballistic to diffusive transport regimes. We have found that the strong noise suppression observed under ballistic conditions remains important also in a wide quasi-ballistic region, before being completely washed out in the diffusive regime. The role played by number and velocity-number fluctuations in the total noise has been quantitatively estimated and found to explain the microscopic origin of the suppression mechanism.

This work has been partially supported by the Comisión Interministerial de Ciencia y Tecnología through the project TIC95-0652.

* Present address: Dept. Física Fonamental, Universitat de Barcelona, Av. Diagonal 647, E-08028 Barcelona, Spain.

[1] Landauer R 1993 Phys. Rev. B 4716427

[2] Reggiani L and Kuhn T 1991 Granular Nanoelectronics (NATO ASI Ser.) ed D K Ferry, J R Barker and C Jacoboni (New York: Plenum) p 287

[3] de Jong M J M and Beenakker C W J 1996 condmat/9611140
[4] Li Y P, Tsui D C, Heremans J J, Simmons J A and Weimann G W 1990 Appl. Phys. Lett 57774

[5] Liefrink F, Dijkhuis J I, de Jong M J M, Molenkamp L W and van Houten H 1994 Phys. Rev. B 4914066

[6] Reznikov M, Heiblum M, Shtrikman H and Mahalu D 1995 Phys. Rev. Lett. 753340

[7] Kumar A, Saminadayar L, Glattli D C, Jin Y and Etienne B 1996 Phys. Rev. Lett. 762778

[8] Steinbach A H, Martinis J M and Devoret M H 1996 Phys. Rev. Lett. 763806

[9] Lesovik G B 1989 JETP Lett. 49594

[10] Beenakker C W J and Büttiker M 1992 Phys. Rev. B 46 1889

[11] de Jong M J M and Beenakker C W J 1995 Phys. Rev. B 5116867

[12] González T, Bulashenko O M, Mateos J, Pardo D and Reggiani L 1997 Phys. Rev. B 56 6424; cond-mat/9703110

[13] We assume elastic isotropic scattering and use an energyindependent $\tau$ for simplicity, but our model can be easily extended to include any inelastic collision with energydependent relaxation time.

[14] González T and Pardo D 1993 J. Appl. Phys. 737453

[15] The linear dependence of the current on $\ell$ in the diffusive regime is a consequence of our model. The choice of an isotropic, elastic scattering with energy-independent $\tau$ leads to ohmic response of the carriers (constant mobility $\mu=q \tau / m)$ at any voltage when transport is dominated by collisions.

FIG. 1. Current-noise spectral density $S_{I}$ vs the ballistic parameter $\ell=v_{\text {th }} \tau / L$ calculated by using static (squares, dashed line) and dynamic (circles, solid line) potentials. The dotted line represents $2 q I$.

FIG. 2. Decomposition of the $S_{I}$ of Fig. 1 into: (a) velocity, (b) number, and (c) velocity-number contributions vs ballistic parameter $\ell=v_{\mathrm{th}} \tau / L$ for the static (squares, dashed line) and dynamic (circles, solid line) potentials. 


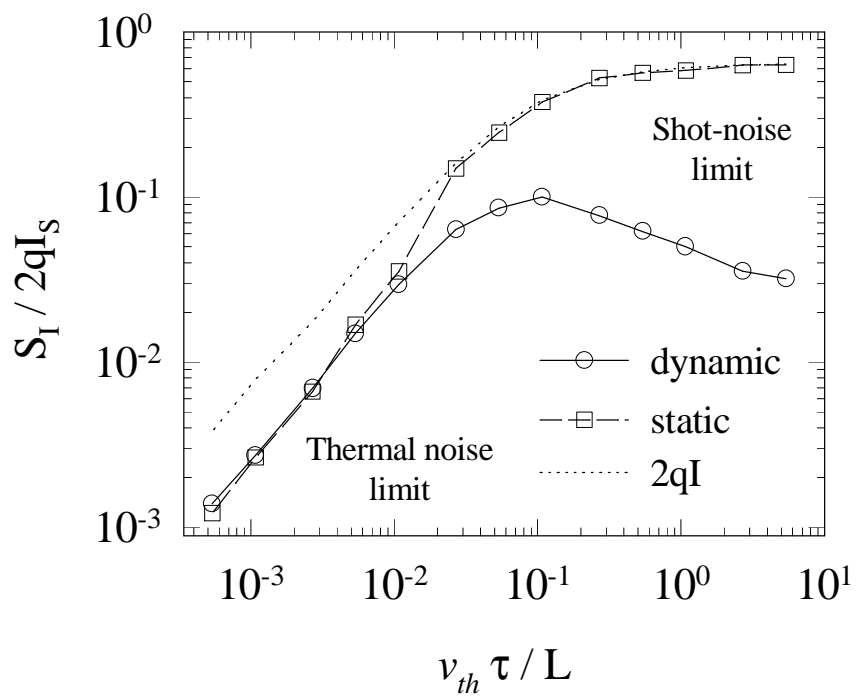

Figure 1

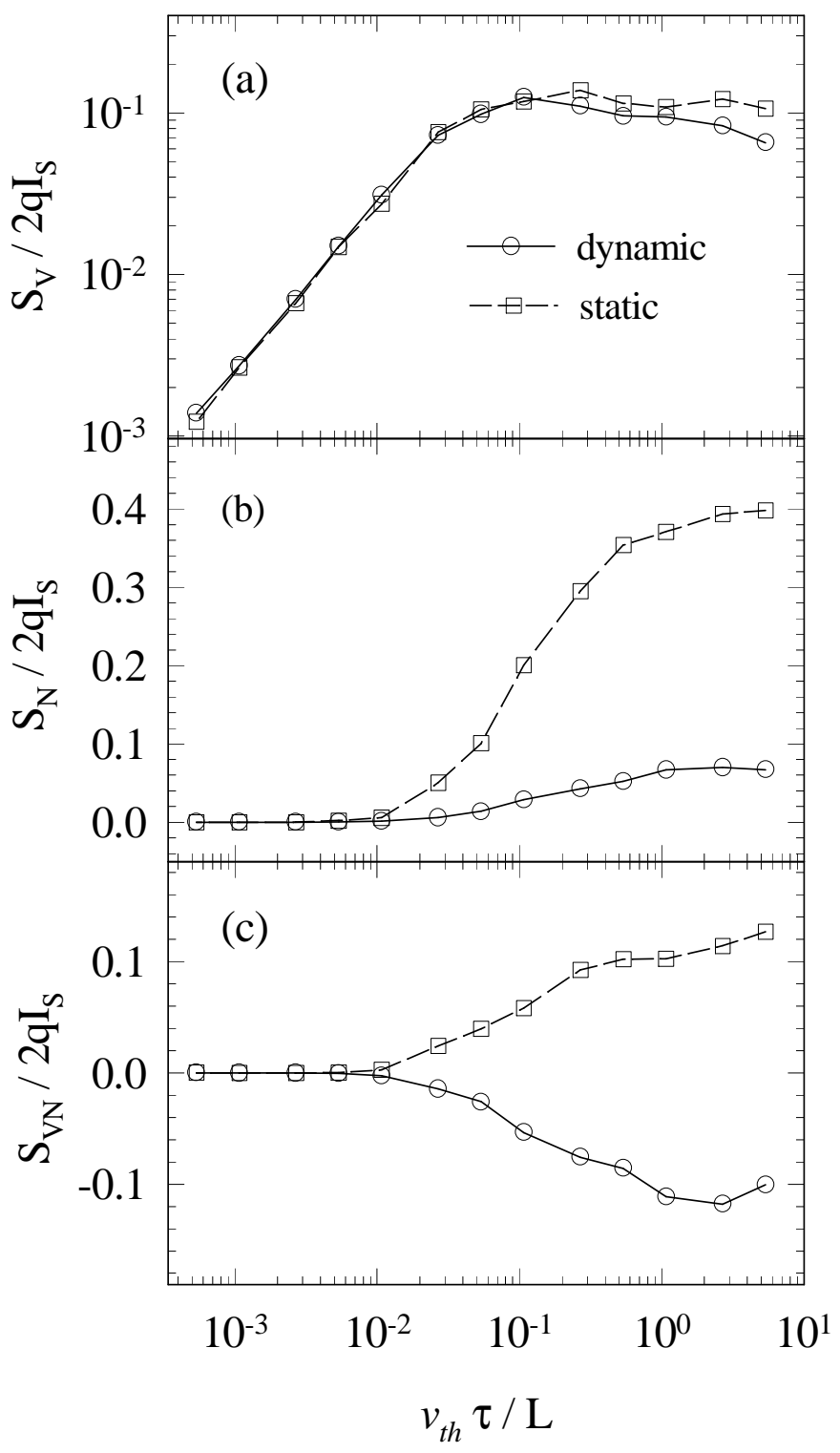

Figure 2 